\title{
Insulin resistance as early sign of hepatic dysfunction in liver cirrhosis
}

\author{
Kayo Taguchi', Hisami Yamanaka-Okumura', Akira Mizuno², Taki Nakamura', \\ Mitsuo Shimada ${ }^{3}$, Toshio Doi ${ }^{2}$, and Eiji Takeda ${ }^{1}$ \\ ${ }^{1}$ Department of Clinical Nutrition, Institute of Health Biosciences, University of Tokushima Graduate \\ School, Tokushima, Japan, ${ }^{2}$ Department of Clinical Biology and Medicine, Institute of Health Bio- \\ sciences, University of Tokushima Graduate School, Tokushima, Japan, ${ }^{3}$ Department of Digestive and \\ Pediatric Surgery, Institute of Health Biosciences, University of Tokushima Graduate School, Tokushima, \\ Japan
}

\begin{abstract}
Glucose intolerance characterized by postprandial hyperglycemia and hyperinsulinemia is commonly seen in patients with liver cirrhosis (LC). The aim of this study is to clarify the relation between glucose intolerance and disorder of liver function in patients with LC. The $75 \mathrm{~g}$ oral glucose tolerance test ( $75 \mathrm{~g}$ OGTT) and the hyperinsulinemic euglycemic clamp combined with $0.2 \mathrm{~g} / \mathrm{kg}$ oral glucose load (HECGL) were conducted in 61 patients with LC. Based on the results of $75 \mathrm{~g}$ OGTT, the 61 patients with LC were divided into groups, 21 (34.4\%) patients with normal glucose tolerance (LC-NGT), 12 (19.7\%) patients with impaired glucose tolerance (LC-IGT) and 28 (45.9\%) patients with diabetes mellitus (LC-DM). Fasting plasma glucose (FPG) level was normal in 50 (82.0\%) patients with LC. All patients with LC showed insulin resistance in both peripheral (skeletal and adipose) and hepatic tissues evaluated by HECGL, although significant correlation between the degree of glucose intolerance and the severity of hepatic dysfunction was not observed. Insulin resistance in both liver and peripheral tissues is the early sign in the patients with LC. This fact indicates that nutritional care from early stages of LC would be necessary in the patients. J. Med. Invest. 61 : 180-189, February, 2014
\end{abstract}

Keywords : nutritional assessment, liver cirrhosis, hyperinsulinemic euglycemic clamp combined with oral glucose load, indirect calorimetry, diabetes mellitus

\section{INTRODUCTION}

The patients with liver cirrhosis (LC) frequently show postprandial hyperglycemia. From 10 to $40 \%$

Abbreviations :

LC : liver cirrhosis, OGTT : oral glucose tolerance test, HECGL : hyperinsulinemic euglycemic clamp combined with $0.2 \mathrm{~g} / \mathrm{kg}$ oral glucose load, NGT : normal glucose tolerance, IGT : impaired glucose tolerance, DM : diabetes mellitus, LC-NGT : liver cirrhosis patients with normal glucose tolerance, LC-IGT : liver cirrhosis patients with impaired glucose tolerance, LC-DM : liver cirrhosis patients with diabetes mellitus, FPG : Fasting plasma glucose, IRI : immunoreactive insulin, $\mathrm{CPR}: \mathrm{C}$ peptide immunoreactivity, $\mathrm{HBV}$ : hepatitis $\mathrm{B}$ virus, $\mathrm{HCV}$ : hepatitis $\mathrm{C}$ virus, $\mathrm{PG}$ : plasma glucose, OGL: oral glucose load, IFG : impaired fasting glucose, of them fall into diabetes mellitus (DM) and about $40 \%$ of them become impaired glucose intolerance (IGT), whereas $30 \%$ of them have kept normal glucose tolerance (NGT) $(1,2)$. It is supposed that

GIR : glucose infusion rate, HGU : hepatic glucose uptake, REE : resting energy expenditure, $\mathrm{npRQ}$ : non-protein respiratory quotient, NEFA : non-esterified fatty acids, AUC : area under the curve

Received for publication December 9, 2013 ; accepted January 22, 2014.

Address correspondence and reprint requests to Mrs. Hisami Yamanaka-Okumura, Department of Clinical Nutrition, Institute of Health Biosciences, University of Tokushima Graduate School, Tokushima 770-8503, Japan and Fax : +81-88-633-7094. 
insulin resistance may contribute to postprandial hyperglycemia since plasma immunoreactive insulin (IRI) and C peptide immunoreactivity (CPR) levels are increased in patients with LC (3). Irrespective of etiology, insulin resistance is observed in the patients with LC even before occurring glucose intolerance (4). Furthermore, diabetes $(5,6)$ or obesity $(5,7)$ characterized by insulin resistance increases cancer risk in cirrhosis.

It has been reported that hepatic glucose production in the patients with LC is normal and decreased (8), however, insulin-induced glucose uptake in the peripheral tissue is markedly decreased (9). In contrast, hepatic insulin resistance is seen in cirrhotic patients with DM but not in patients with LC alone (9-11). Thus, the role of insulin resistance on hepatic glucose uptake in the patients with LC has remained unclear.

There was no clear evidence that etiology, duration and degree in the patients with LC would be related to glucose intolerance (12). Furthermore, disorder in glucose metabolism is not always seen in a comparative number of cirrhotic patients and the patients with advanced LC is not always developed IGT and DM (12).

In this study, the relation between glucose uptake of peripheral and liver tissue evaluated by the hyperinsulinemic euglycemic clamp combined with 0.2 $\mathrm{g} / \mathrm{kg}$ oral glucose load (HECGL), and the biochemical and metabolic parameters of liver function was examined in the patients with $\mathrm{LC}$ divided into groups by World Health Organization criteria in $75 \mathrm{~g}$ oral glucose tolerance test (OGTT) (13). HECGL (1416) which enabled us to measure the peripheral and hepatic glucose uptake simultaneously and precisely was conducted in 61 patients with LC.

\section{SUBJECTS AND METHODS}

\section{Subjects}

Sixty-one patients with LC (16 hepatitis B virus (HBV)-infected LC, 35 hepatitis $\mathrm{C}$ virus (HCV)infected LC, 3 HBV- and HCV-infected LC, 2 alcoholic LC and 5 LC with unknown etiology) and 34 age matched control without liver disease (Control) were enrolled for this study. None of the patients received any medication affecting glucose tolerance, including oral hypoglycemic agents or insulin injections. All patients with LC were diagnosed by liver biopsy, laboratory tests and ultrasound sonography ; alcoholic liver disease was diagnosed in habitual drinkers who consumed more than $30 \mathrm{~g}$ of ethanol daily. None of the patients had ascites, jaundice or hepatic encephalopathy. 48 and 13 patients in $61 \mathrm{LC}$ patients were classified as Child-Pugh A and B, respectively. The clinical and laboratory data are summarized in Table 1 . The levels of white blood cell, platelet, albumin, cholinesterase, total cholesterol and triglyceride in the patients with LC were significantly lower than those in Control ; body mass index and the levels of serum aspartate aminotransferase,

Table 1. Clinical and laboratory data of age matched control and patients with liver cirrhosis

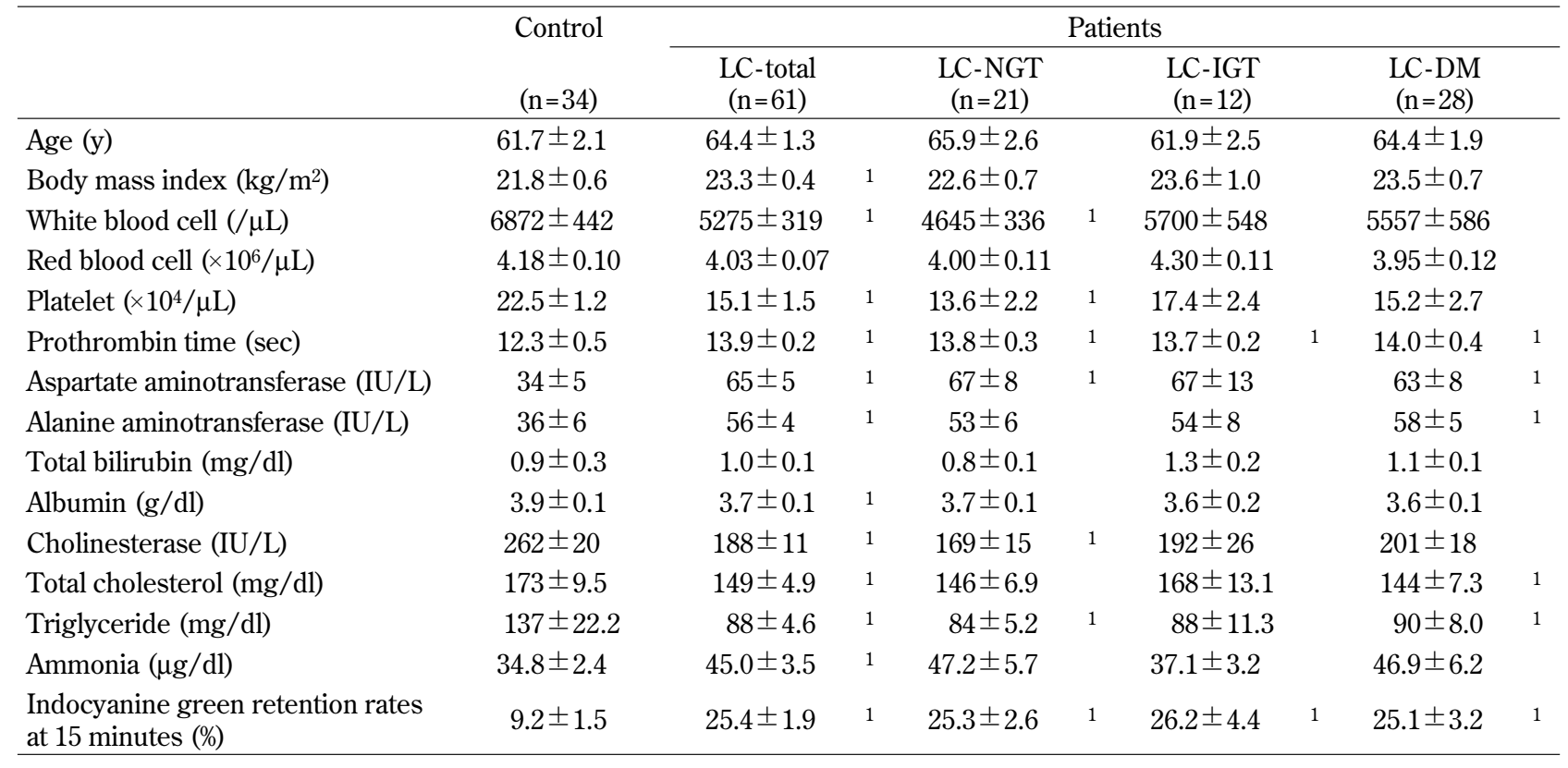

All values are mean \pm SE. ${ }^{1} \mathrm{p}<0.05$ vs. Control 
alanine aminotransferase and ammonia, prothrombin time, and indocyanine green retention rates at 15 minutes were significantly higher than those in Control.

The purpose of the study was fully explained to all subjects, and their informed and written consent were obtained. The study confirmed to the 1975 Helsinki Declaration on ethical guidelines for clinical investigations.

\section{Oral glucose tolerance test}

After overnight fasting for $12 \mathrm{~h}$, the patients ingested $75 \mathrm{~g}$ of dextrose (Torelan G 75, Shimizu Co., Japan) within 5 min. Venous blood was drawn for measuring plasma glucose (PG), IRI and CPR levels before, and at 15, 30, 60 and 120 min after the oral glucose load (OGL). The definition of DM, impaired fasting glucose (IFG), IGT and NGT were based on World Health Organization criteria (13). In brief, patients were classified as LC-DM when fasting plasma glucose (FPG) level was $\geqq 126 \mathrm{mg}$ / $\mathrm{dl}$ or when PG level at 2-hour OGTT was $\geqq 200 \mathrm{mg} /$ dl. LC-IGT was defined as FPG level of $<126 \mathrm{mg}$ / $\mathrm{dl}$ and 2-hour OGTT PG level of 140 to $199 \mathrm{mg} / \mathrm{dl}$. Thus, patients with LC-IFG had FPG level of 110 to $125 \mathrm{mg} / \mathrm{dl}$ and 2-hour OGTT PG level of $<140 \mathrm{mg} /$ dl. Patients with LC-NGT had FPG level of $<110$ $\mathrm{mg} / \mathrm{dl}$ and 2 -hour OGTT PG level of $<140 \mathrm{mg} / \mathrm{dl}$.

\section{Hyperinsulinemic euglycemic clamp combined with oral glucose load}

Subjects were admitted to the laboratory at 8:00AM after an overnight fast. They voided, were weighed, and then remained supine for the duration of the procedure. The details of HECGL have been described previously (14-16). In short, a polyethylene catheter was inserted into an antecubital vein in a retrograde manner to administer glucose (20\% of glucose) and insulin infusion. A second catheter was inserted into a dorsal hand vein on the contralateral arm in the retrograde fashion and kept in a warming device to arterialize the venous blood. Blood samples were drawn at baseline to determine Fasting PG levels, serum IRI levels and CPR levels. Insulin-mediated whole body glucose uptake was measured using an artificial pancreas (Model STG22 Nikkiso, Tokyo, Japan), according to DeFronzo's method (17). An infusion of $20 \%$ of glucose solution was started at baseline, and the rate was adjusted to clamp the glucose level at $100 \mathrm{mg} / \mathrm{dl}$ during the clamp study. PG levels were checked at 30 -second intervals throughout the investigation. Data on total body glucose uptake represented the mean values for the glucose infusion rate (GIR) during the final 30 minutes of infusion. After determining the baseline GIR during the hyperinsulinemic euglycemic clamp described above, glucose was orally administered at a dose of $0.2 \mathrm{~g} / \mathrm{kg}$ body weight. Thereafter, the hyperinsulinemic euglycemic clamp was continued and the extent of decrease in GIR was monitored for $90 \mathrm{~min}$ in order to evaluate hepatic glucose uptake (HGU), which was used as a parameter of insulin sensitivity in the liver.

\section{Calculation of hepatic glucose uptake}

After an OGL during hyperinsulin emiceuglycemic clamp, the glucose appearance rate $(\mathrm{Ra}(\mathrm{t}))$ is calculated by the formula,

$\mathrm{Ra}(\mathrm{t})=[\mathrm{OGL}(\mathrm{t})-\mathrm{HGU}(\mathrm{t})]+\mathrm{GIR}(\mathrm{t})$ [1] where OGL $(\mathrm{t})$, the rate of absorption of OGL by the intestine at time $t ; \mathrm{HGU}(\mathrm{t})$, the rate of HGU of OGL at time $t$; and GIR(t), GIR at time $t$. Glucose disappearance rate from the systemic circulation $(\operatorname{RdT}(t))$ is calculated by the formula,

$\operatorname{RdT}(\mathrm{t})=\operatorname{RdP}(\mathrm{t})+\operatorname{RdS}(\mathrm{t})$ where $\operatorname{RdP}(t)$ and $\operatorname{RdS}(t)$ are extra-splanchnic and splanchnic glucose disappearance rates from systemic circulation at time $t$, respectively. Under hyperinsulinemic euglycemic clamp conditions, $\mathrm{Ra}(\mathrm{t})$ is equal to $\operatorname{RdT}(\mathrm{t})$, therefore

$\operatorname{RdP}(\mathrm{t})+\operatorname{RdS}(\mathrm{t})=[\mathrm{OGL}(\mathrm{t})-\mathrm{HGU}(\mathrm{t})]+\mathrm{GIR}(\mathrm{t})-[3]$ HGU can be calculated from the difference between the amount of ingested glucose and the summation of GIR decrements after glucose ingestion. $\Sigma(\operatorname{HGU}(\mathrm{t})+\operatorname{RdS}(\mathrm{t}))=\Sigma \mathrm{OGL}(\mathrm{t})-\Sigma(\mathrm{RdP}(\mathrm{t})-\mathrm{GIR}(\mathrm{t}))$

Total hepatic glucose uptake was expressed as a percentage of OGL.

\section{Assessment of energy metabolism}

To maintain the resting energy expenditure (REE) and substrate oxidation, in preprandial and postprandial thermogenesis after an overnight fast, the patients and Control were asked to maintain a supine position throughout the study period, and to avoid activity during the indirect calorimetry (Chest MI, Tokyo, Japan). The measurements of $\mathrm{O}_{2}$ consumption and $\mathrm{CO}_{2}$ production were computed and printouts obtained at $1 \mathrm{~min}$ intervals ; the measurements were continued until steady-state equilibrium was achieved. The average $\mathrm{O}_{2}$ consumption and $\mathrm{CO}_{2}$ production were used in calculating the energy expenditure ; the mean values during the last $10 \mathrm{~min}$ of the measurement interval were used for calculation. 
The machine was calibrated before each study and at intervals throughout the experiment. The basal energy expenditure was estimated according to the equation reported by Harris and Benedict (18), and the ratio of REE to basal energy expenditure is expressed as the \%REE. The \%REE was used as the actual index of energy expenditure to compare the values of energy expenditure among different individuals. The non-protein respiratory quotient (npRQ) was calculated from measurements of daily urinary nitrogen excretion. Measurements were taken at $8: 30 \mathrm{AM}, 11: 30 \mathrm{AM}$ and $2: 30 \mathrm{PM}$. The patients were asked to stay in bed for at least 30 minutes before each measurement, but some mobility was allowed between each set of measurements. Breakfast and lunch were given at $9: 00 \mathrm{AM}$ and $12: 00$ noon, respectively, and consumed within $30 \mathrm{~min}$. $\mathrm{Pa}-$ tients received standard hospital food, and their dietary intake was recorded and the amounts of energy, protein, fat, and carbohydrate were calculated using Japanese standard tables of food composition (19). The energy and nutritional intake was not significantly different among the groups. Blood samples were taken to determine the PG and non-esterified fatty acids (NEFA) just before the indirect calorimetry measurements.

\section{Measurement and statistical analysis}

Glucose was measured by the glucose oxidase method (Hitachi 736 Auto Analyser, Hitachi Co.,
Tokyo, Japan). IRI and CPR were analyzed by radioimmunoassay (Eiken, Tokyo, Japan). Serum NEFA concentrations were assayed using an acyl-CoA oxidase-based colorimetric kit (Wako NEFA-C, Wako Chemicals, Osaka, Japan). All data were presented as mean $\pm \mathrm{SE}$, unless otherwise specified. Statistical comparisons were assessed, where appropriate, using one-way ANOVA and unpaired t-test analysis. $P$ values $<0.05$ were considered statistically significant.

\section{RESULTS}

1) Classification of the patients with LC by $75 \mathrm{~g}$ OGTT

By $75 \mathrm{~g}$ OGTT, the 61 patients with LC were divided into $21(34.4 \%)$ in LC-NGT, 12 (19.7\%) in LCIGT, 28 (45.9\%) in LC-DM, but there were no patients in LC-IFG (Figure 1). FPG level was normal in $50(82.0 \%)$ patients with LC. PG in LC-total patients was higher than that in Control at 15, 30, 60 and $120 \mathrm{~min}$. There was not significant difference between Control and LC-DM patients, but PG in LC-DM patients was higher than that in LC-NGT patients and LC-IGT patients at fasting state. Postprandial PG in LC-DM patients was higher than that in LC-NGT patients at 30, 60, 120 min and that in LC-IGT patients at $120 \mathrm{~min}$, respectively. IRI in LCtotal patients was higher than that in Control at 60
(A)

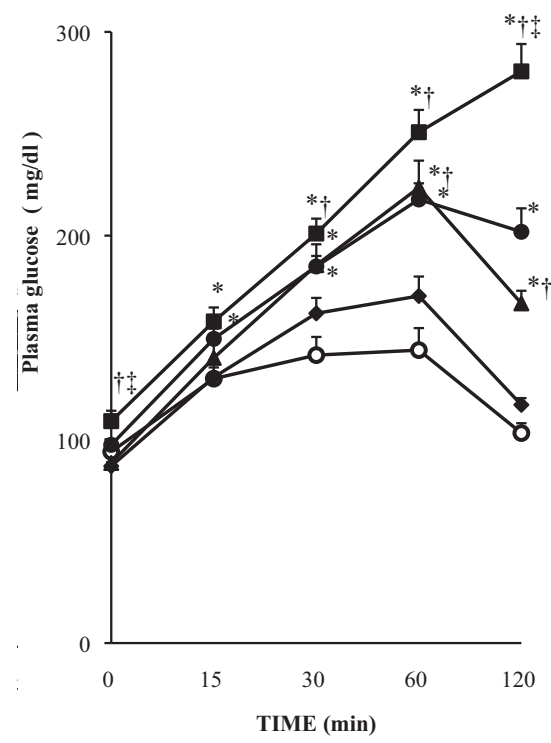

(B)

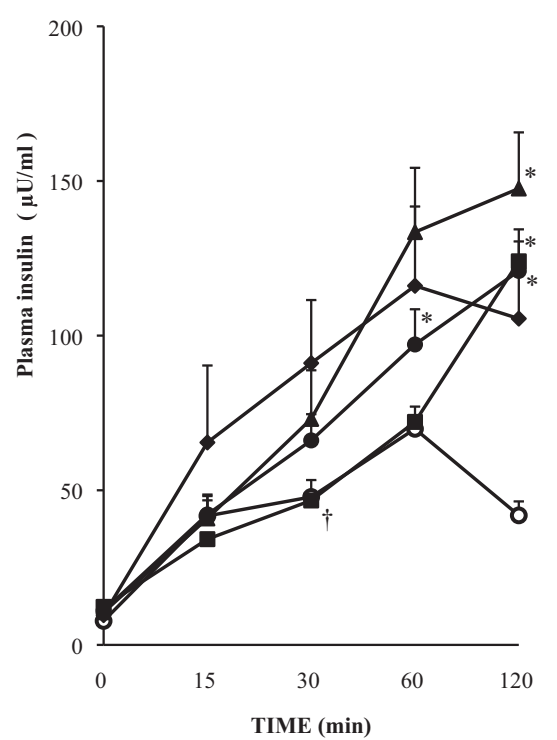

(C)

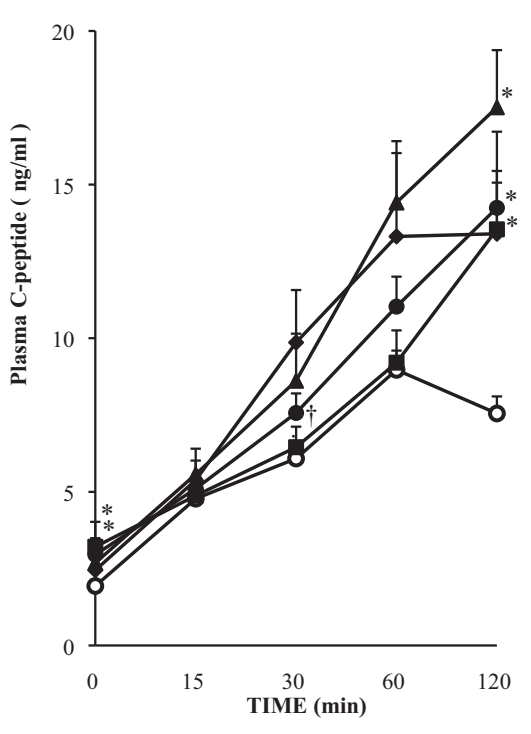

Figure 1. Plasma glucose (A), insulin (B), and C-peptide (C), levels during $75 \mathrm{~g}$ oral glucose tolerance test in control $(\bigcirc)$ and patients with liver cirrhosis (LC) total $(\bullet)$, NGT: normal glucose tolerance $(\bullet)$, IGT: impaired glucose tolerance $(\boldsymbol{\Lambda})$, DM : diabetes

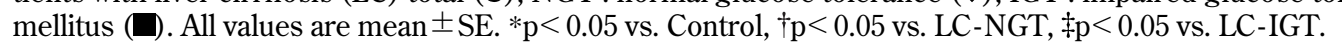


and $120 \mathrm{~min}$. There was not significant difference between Control and LC-total patients at fasting state. The Insulin level at fasting state tended to be higher with insulin resistance, but it was not significant. IRI in LC-DM patients was lower than that in LC-NGT patients at $30 \mathrm{~min}$. Similarly, CPR in LCDM patients was lower than that in LC-NGT patients at $30 \mathrm{~min}$. CPR in LC-total patients was higher than that in Control at 0 and $120 \mathrm{~min}$. The values of area under the curve (AUC) of PG, IRI and CPR levels in LC-total patients were significantly higher than those in Control. The AUC of PG in LC-DM patients higher than that in Control, LC-NGT patients and LC-IGT patients. That in LC-IGT patients higher than that in Control and LC-NGT patients.
The AUC of CPR in LC-IGT patients higher than that in Control (Figure 2).

The ratios of AUC of IRI to AUC of CPR (AUC IRI/AUC CPR), a rough measurement for porto-systemic shunting of insulin, in LC-total patients, LCNGT patients, LC-IGT patients and LC-DM patients tended to be higher than those in Control, but not significant.

\section{2) Evaluation of insulin sensitivity}

Comparable and stable PG levels, clamped at 1.00 $\mathrm{g} / \mathrm{L}$, were achieved over the last $30 \mathrm{~min}$ in patients with LC and Control with HECGL. Both GIR and HGU in LC-total patients were significantly lower than those in Control (Figure 3). GIR in LC-NGT,
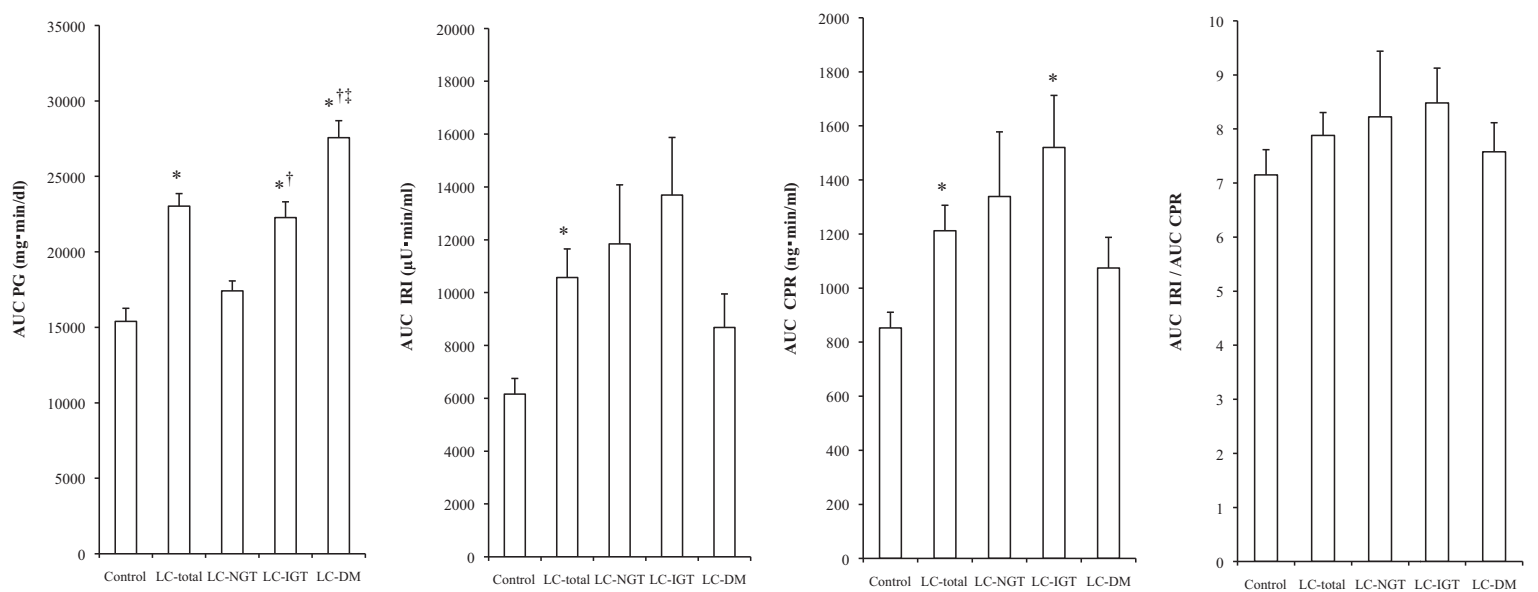

Figure 2. Area under the curve of plasma glucose (PG), immunoreactive insulin (IRI) and C-peptide immunoreactivity (CPR) for $120 \mathrm{~min}$ after $75 \mathrm{~g}$ oral glucose tolerance test in control and patients with liver cirrhosis. All values are mean \pm SE. AUC : area under the curve, LC : liver cirrhosis, NGT : normal glucose tolerance, IGT : impaired glucose tolerance, DM : diabetes mellitus. $* \mathrm{p}<0.05 \mathrm{vs}$. Control, $\dagger \mathrm{p}<0.05$ vs. LC-NGT, $\neq \mathrm{p}<0.05$ vs. LC-IGT.
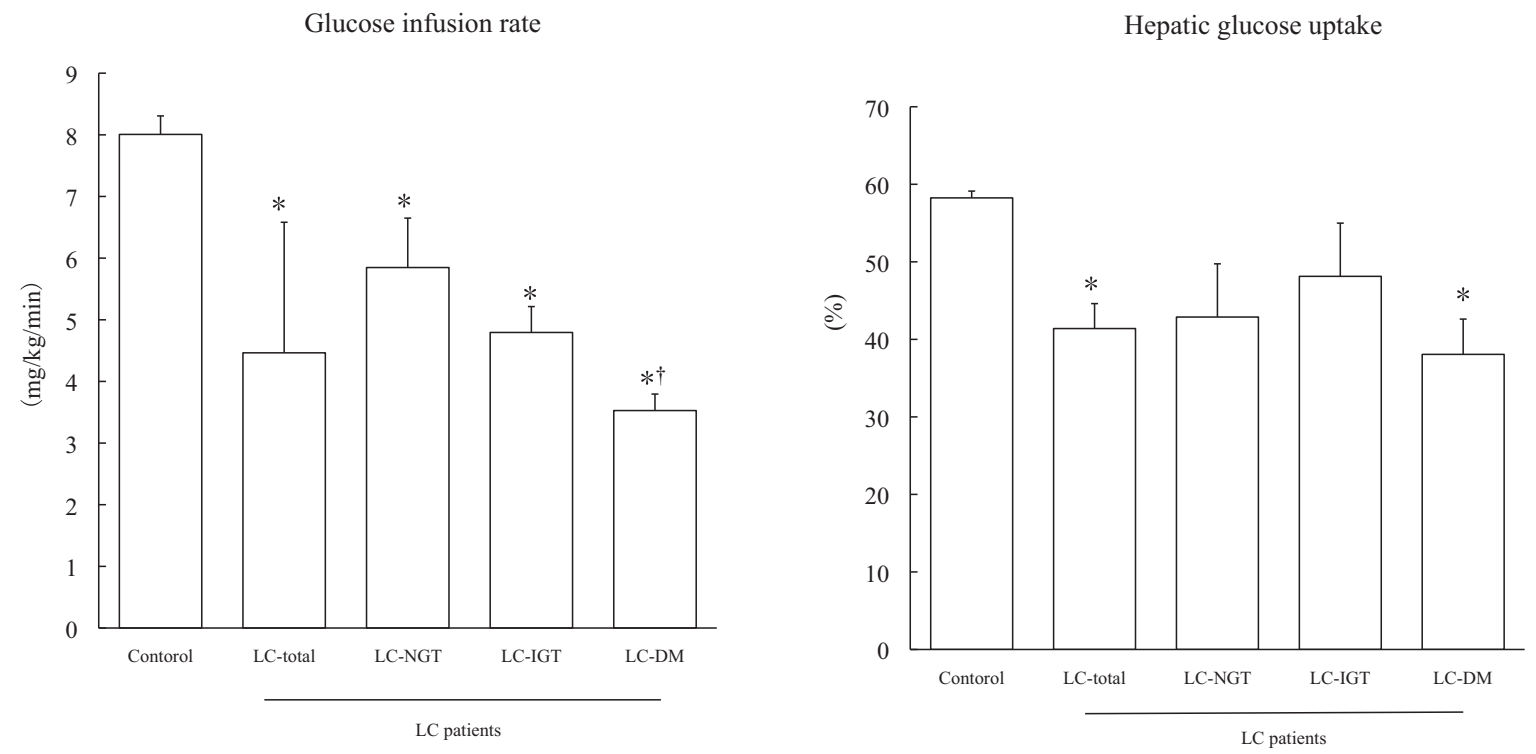

Figure 3. Glucose infusion rate and hepatic glucose uptake by hyperinsulinemic euglycemic clamp combined with oral glucose load in control and patients with liver cirrhosis. All values are mean \pm SE. LC : liver cirrhosis, NGT : normal glucose tolerance, IGT: impaired glucose tolerance, DM : diabetes mellitus. $* \mathrm{p}<0.05$ vs. Control, $\dagger \mathrm{p}<0.05$ vs. LC-NGT. 
LC-IGT and LC-DM patients showed significantly lower than that in Control. Furthermore, GIR in LCDM patients showed significantly lower than that in LC-NGT patients. Thus, GIR levels proportionally became lower with the severity of glucose intolerance. HGU in LC-DM patients showed significantly lower than that in Control.

\section{3) Comparison of biochemical and metabolic fea- tures in LC patients}

Age was not significant differences among each group. Blood chemistry data exhibited significant differences between Control and LC patients (Table 1). In energy metabolism, the \%REE values in the patients with LC and those groups were similar to those of Control under fasting and postprandial conditions (Table 2). Fasting npRQ in LC-total patients was significantly lower than that in Control, and the value tended to decrease according to the severity of glucose intolerance in the patients with LC. Furthermore, Fasting npRQ in LC-DM patients showed significantly lower than that in Control. Such findings were not observed after food ingestion. Therefore, the increased npRQ levels after meal, designated as $\mathrm{npRQ}$, in the patients with $\mathrm{LC}$ were higher than those in Control, and the value tended to increase according to the severity of glucose intolerance in the patients with LC. Furthermore, $\Delta \mathrm{npRQ}$ in LC-DM patients showed significantly higher than that in Control. Fasting and postprandial glucose concentrations in LC-total patients were significantly higher than those in Control. PG in LC patients, at $11: 30 \mathrm{AM}$ and $2: 30 \mathrm{PM}$, were elevated with the severity of glucose intolerance, which was similar to those of $75 \mathrm{~g}$ OGTT. The serum fasting NEFA concentrations in LC-total patients were significantly higher than those in Control and tended to increase with the severity of impaired glucose metabolism in patients with LC. Furthermore, fasting NEFA in LC-DM patients showed significantly higher than that in Control.

\section{DISCUSSION}

In this study, we did not find that there were certainly correlation between degree of glucose intolerance and severity of liver dysfunction in the patients with LC. It has been reported that incidence of glucose intolerance in the patients with LC was $60-80 \%$ and defined DM was $10-60 \%(1,2,20)$.

To verify how many prevalence of glucose intolerance was observed in the patients with $\mathrm{LC}, 75 \mathrm{~g}$ OGTT was firstly conducted in this study. The data in OGTT were compared with those in Control. In this study, the prevalence of NGT, IGT and DM was $34 \%, 20 \%$ and $46 \%$, respectively. Hepatic glucose uptake and peripheral glucose uptake were measured simultaneously using HECGL. The validity of this method for evaluating HGU had been ascertained

Table 2. Effects on meal ingestion on \%REE, npRQ, $\triangle \mathrm{npRQ}$ and the concentration of plasma glucose (PG) and serum NEFA in control and patients with liver cirrhosis

\begin{tabular}{|c|c|c|c|c|c|c|c|c|c|}
\hline & & \multirow{2}{*}{ Control } & \multicolumn{7}{|c|}{ Patients } \\
\hline & & & LC-total & & LC-NGT & LC-IGT & & LC-DM & \\
\hline$\% \mathrm{REE}$ & $8: 30 \mathrm{AM}$ & $93.3 \pm 1.8$ & $94.6 \pm 1.5$ & & $95.8 \pm 3.1$ & $99.1 \pm 2.7$ & & $92.6 \pm 2.1$ & \\
\hline \multirow[t]{2}{*}{$(\%)$} & $11: 30 \mathrm{AM}$ & $102.8 \pm 2.8$ & $105.6 \pm 1.8$ & & $107.9 \pm 3.9$ & $111.6 \pm 4.4$ & & $102.1 \pm 2.0$ & \\
\hline & $2: 30 \mathrm{PM}$ & $109.2 \pm 3.0$ & $113.7 \pm 2.4$ & & $113.9 \pm 4.0$ & $123.1 \pm 8.2$ & & $110.0 \pm 2.4$ & \\
\hline \multirow[t]{3}{*}{$\mathrm{npRQ}$} & $8: 30 \mathrm{AM}$ & $0.801 \pm 0.016$ & $0.753 \pm 0.009$ & 1 & $0.785 \pm 0.017$ & $0.763 \pm 0.028$ & & $0.732 \pm 0.010$ & 1 \\
\hline & $11: 30 \mathrm{AM}$ & $0.847 \pm 0.016$ & $0.870 \pm 0.011$ & & $0.896 \pm 0.022$ & $0.877 \pm 0.036$ & & $0.853 \pm 0.011$ & \\
\hline & $2: 30 \mathrm{PM}$ & $0.866 \pm 0.019$ & $0.889 \pm 0.120$ & & $0.913 \pm 0.014$ & $0.894 \pm 0.042$ & & $0.873 \pm 0.162$ & \\
\hline \multirow[t]{2}{*}{$\Delta \mathrm{npRQ}$} & $11: 30 \mathrm{AM}-8: 30 \mathrm{AM}$ & $0.048 \pm 0.015$ & $0.118 \pm 0.013$ & 1 & $0.111 \pm 0.021$ & $0.114 \pm 0.046$ & & $0.124 \pm 0.018$ & 1 \\
\hline & $2: 30 \mathrm{PM}-8: 30 \mathrm{AM}$ & $0.067 \pm 0.014$ & $0.136 \pm 0.155$ & 1 & $0.128 \pm 0.025$ & $0.131 \pm 0.053$ & & $0.142 \pm 0.020$ & 1 \\
\hline \multirow[t]{3}{*}{$\mathrm{PG}(\mathrm{mg} / \mathrm{dl})$} & $8: 30 \mathrm{AM}$ & $88.7 \pm 1.8$ & $107.0 \pm 4.1$ & 1 & $94.0 \pm 2.0$ & $92.3 \pm 4.5$ & & $120.0 \pm 6.7$ & $1,2,3$ \\
\hline & $11: 30 \mathrm{AM}$ & $113.6 \pm 5.3$ & $153.7 \pm 8.9$ & 1 & $119.0 \pm 10.6$ & $125.3 \pm 8.8$ & & $187.6 \pm 13.0$ & $1,2,3$ \\
\hline & $2: 30 \mathrm{PM}$ & $110.7 \pm 4.0$ & $142.4 \pm 9.3$ & 1 & $115.2 \pm 5.6$ & $136.1 \pm 19.1$ & & $163.0 \pm 16.1$ & 1,2 \\
\hline \multirow[t]{3}{*}{$\operatorname{NEFA}(\mu \mathrm{Eq} / \mathrm{L})$} & $8: 30 \mathrm{AM}$ & $434.7 \pm 27.7$ & $570.6 \pm 29.9$ & 1 & $503.5 \pm 53.7$ & $548.4 \pm 67.0$ & & $619.5 \pm 41.4$ & 1 \\
\hline & $11: 30 \mathrm{AM}$ & $138.1 \pm 18.0$ & $124.9 \pm 12.8$ & & $122.8 \pm 21.3$ & $83.0 \pm 6.2$ & 1 & $138.8 \pm 20.2$ & \\
\hline & $2: 30 \mathrm{PM}$ & $111.3 \pm 7.9$ & $107.5 \pm 9.4$ & & $103.5 \pm 14.3$ & $62.6 \pm 6.4$ & 1 & $125.3 \pm 14.4$ & 3 \\
\hline
\end{tabular}

All values are mean \pm SE. \%REE $: \%$ of resting energy expenditure to basal energy expenditure, $\mathrm{npRQ}:$ nonprotein respiratory quotient, $\triangle \mathrm{npRQ}$ : changes of $\mathrm{npRQ}, \mathrm{BG}$ : blood glucose, NEFA : non esterified fatty acid

NGT : normal glucose tolerance, IGT : impaired glucose tolerance, DM : diabetes mellitus ${ }^{1} \mathrm{p}<0.05$ vs. Control, ${ }^{2} \mathrm{p}<0.05$ vs. LC-NGT, ${ }^{3} \mathrm{p}<0.05$ vs. LC-IGT 
by the hepatic vein catheterization procedure in humans (15) and in dogs (21). In the present study, GIR in the patients with LC was significantly lower than that in normal subjects. Furthermore, HGU in them was about $70 \%$ of that in Control. GIR decreased depend on severity of glucose intolerance. But HGU did not show significant differences among LC groups. i.e., severity of glucose intolerance was seen to correlate with GIR but not HGU.

Recent studies using the hyperinsulinemic euglycemic glucose clamp technique have reported that peripheral insulin sensitivity in the patients with LC was about $30-60 \%$ of that in healthy volunteers $(9,10)$. The study using a positron-emission tomography study has clarified that in the patients with LC impaired insulin action in the peripheral tissues is characterized by both marked decrease in glucose transport and nonoxidative glucose metabolism under euglycemic state, however, glucose phosphorylation and glucose oxidation have been kept to be intact (22). In addition, decreased insulin-induced glycogen synthase activity was demonstrated in isolated muscle samples from the patients with LC (23).

More than $65 \%$ of the patients with LC show protein-energy malnutrition because of their increased fat oxidation and decreased glucose oxidation (24). It was reported that fat oxidation rate measured in starved state after an overnight fast was increased because glycogen stores in the patients with LC was depleted in this condition (24). The nutritional condition is frequently reduced and about $70 \%$ of the patients with LC show some signs of malnutrition (25). It is well recognized that malnutrition contributes to insulin resistance in the patients with LC $(4,26)$. Furthermore, lipotoxicity by NEFA cause insulin resistance $(27,28)$. With respect to REE, there was no significant difference in REE between Control and LC patients. As patients with LC have been reported to have either increased (29), normal (30, 31 ), or decreased (32) resting metabolic rates, this issue remains controversial. The variability observed in the patients with LC, although there was no significant difference in REE between Control and the patients with $\mathrm{LC}$. Whenever available, indirect calorimetry should be used to measure REE in the patients with LC.

The mass and function of hepatocytes is reduced in the cirrhotic liver. Collagen accumulation in the Disse's space may disturb glucose permission from endothelial cell of portal vein to hepatocytes $(33,34)$. It also reported that insulin resistance is associated with more rapid fibrosis progression in chronic $\mathrm{HCV}$ infection $(35,36)$. Therefore, it is supposed that complication with DM indicates the advance in liver damage. The relation between glucose intolerance and the biochemical parameters in liver dysfunction, degree of cirrhosis and energy metabolism in the patients with LC was not observed. The average of estimated HGU in the patients with LC and normal subjects were $41.4 \%$ and $58.3 \%$ in HECGL study, respectively. Previous paper showed HGU was lower in LC patients than in chronic active hepatitis patients and healthy volunteers (37). HGU did not decrease depend on severity of glucose intolerance among LC groups. Thinking about severity of glucose tolerance in LC, but HGU showed significantly lower in LC patients than that in Control. Therefore, our data indicated that postprandial glucose uptake of the liver has already decreased in the patients with LC.

The characteristics of glucose intolerance in the patients with LC are unusual. Although some LC patients exhibit fasting hypoglycemia, they also have postprandial hyperglycemia and continuous hyperinsulinemia (37). Our results also showed that postprandial hyperglycemia was observed in the patients with LC despite fasting glucose levels were normal. In general, insulin resistance is considered to be composed of the following three metabolic defects : 1) reduced glucose uptake by the peripheral tissues ; 2) decreased splanchnic glucose uptake ; 3) overproduction of glucose by the liver. Several studies showed that peripheral and splanchnic glucose utilization is known to be impaired as a result of reduced nonoxidative glucose metabolism in the patients with LC (i.e., glycogen synthesis) (22, 37). Therefore, the main factor that contributes to postprandial hyperglycemia may be associated with insulin resistance.

It seemed that postprandial hyperinsulinemia may overcome peripheral insulin resistance in the patients with LC (38) so that NGT in OGTT was observed in $30 \%$ of patients with LC. Therefore, postprandial hyperglycemia might be one of other compensatory mechanism to disorders in glucose metabolism in the patients with $\mathrm{LC}$, so that the overall glucose disposal is normal in some patients with LC. These findings suggest that glucose intolerance may be initiating event for indicating liver dysfunction and/or altered hepatic circulation in the patients with LC at early stage.

Peripheral insulin resistance characterized by decreased glucose transport and reduced glycogen 
synthesis in skeletal muscle, whereas the insulininduced increases in glucose phosphorylation, glycolysis and glucose oxidation the patients with LC were reported $(4,22,23,39)$. Though, increased insulin level cannot ameliorate disturbed glycogen synthesis and disproportional increase in lactate production in the patients with LC (39). However, glucose transport, glucose phosphorylation, glycolysis, glucose oxidation and glycogen synthesis are all impaired in the patients with type 2 DM (40). Furthermore, it has been reported that further elevation of insulin level can normalize glycogen synthesis and glycolysis but not glucose oxidation and anaerobic glycolysis in the patients with DM (40). Thus, changes in metabolic characteristics in the cirrhotic patients complicated with DM were obvious. In contrast, impaired insulin extraction and impaired counter regulation based on reduced glycogen store in liver tissue and abnormal glucagon secretion (41). These facts suggest that the cirrhotic patients complicated with DM will be fallen into hypoglycemia.

We concluded from the present study that insulin resistance in both liver and peripheral tissues is the early etiology of metabolic abnormality in the patients with LC and precious nutritional care will be needed at early stage of LC.

\section{CONFLICT OF INTEREST}

None of the authors have any conflicts of interest to declare.

\section{ACKNOWLEDGEMENTS}

This publication is based on work supported by Grant-in-Aid for Scientific Research from the Ministry of Education, Science, and Culture, Japan. We wish to thank the doctors and nurses in the Department of Digestive and Pediatric Surgery, Tokushima University Hospital, for their help and cooperation during the study.

\section{REFERENCES}

1. Megysi C, Samols E, Marks V: Glucose tolerance and diabetes in chronic liver disease. Lancet 2 : 1051-1055, 1967

2. Müller MJ, Böker KH, Selberg O : Metabolism of energy-yielding substrates in patients with liver cirrhosis. Clin Investig 72 : 568-579, 1994

3. Nygren A, Adnev N, Sundblad L : Insulin uptake by the human alcoholic liver. Metabolism $34: 48-52,1985$

4. Muller MJ, Willmann O, Rieger A, Fenk A, Selberg O, Lautz HU, Bürger M, Balks HJ, von zur Mühlen A, Schmidt FW : Mechanism of insulin resistance associated with liver cirrhosis. Gastroenterology 102 : 2033-2041, 1992

5. Muto Y, Sato S, Watanabe A, Moriwaki H, Suzuki K, Kato A, Kato M, Nakamura T, Higuchi K, Nishiguchi S, Kumada H, Ohashi Y, Long-Term Survival Study (LOTUS) Group : Overweight and obesity increase the risk for liver cancer in patients with liver cirrhosis and long-term oral supplementation with branchedchain amino acid granules inhibits liver carcinogenesis in heavier patients with liver cirrhosis. Hepatol Res 35 : 204-217, 2006

6. Kasuga M, Ueki K, Tajima N, Noda M, Ohashi K, Noto H, Goto A, Ogawa W, Sakai R, Tsugane S, Hamajima N, Nakagama H, Tajima K, Miyazono K, Imai K : Report of the Japan Diabetes Society/Japanese Cancer Association Joint Committee on Diabetes and Cancer. Cancer Sci 104 : 965-976, 2013

7. Calle EE, Rodriguez C, Walker-Thurmond K, Thun MJ : Overweight, obesity, and mortality from cancer in a prospectively studied cohort of U.S. adults. N Engl J Med 348 : 1625-38, 2003

8. Cavallo-Perin P, Cassader M, Bozzo C, Bruno A, Nuccio P, Dall'Omo AM, Marucci M, Pagano G : Mechanism of insulin resistance is human in human liver cirrhosis. J Clin Invest $75: 1659-1665,1985$

9. Shmueli E, Walker M, Alberti G, Record CO : Normal splanchnic but impaired peripheral insulin-stimulated glucose uptake in cirrhosis. Hepatology 18 : 86-95, 1993

10. Petrides AS, Groop LC, Riely CA, De Fronzo RA : Effect of physiologic hyperinsulinemia on glucose and lipid metabolism in cirrhosis. J Clin Invest $88: 561-570,1991$

11. Petrides AS, Vogt C, Schulze-Berge D, Matthews D, Strohmeyer G : Pathogenesis of glucose intolerance and diabetes mellitus in cirrhosis. Hepatology 19 : 619-627, 1994

12. Muller MJ, Pirlich M, Balks HJ, Selberg O : Glucose intolerance in liver cirrhosis : Role of hepatic and non-hepatic influences. Eur J 
ClinChemClinBiochem $32:$ 749-758, 1994

13. Alberti KG, Zimmet PZ : Definition, diagnosis and classification of diabetes mellitus and its complications. Part1 : diagnosis and classification of diabetes mellitus provisional report of a WHO Consultation. Diabet Med 15 : 539-553, 1998

14. Kawamori R, Morishima T, Ikeda M, Kubota M, Kishimoto M, Shiba Y, Matsuhisa M, Kodama M, Watarai T : Effect of strict metabolic control on glucose handling by the liver and peripheral tissues in non-insulin-dependent diabetes mellitus. Diabetes Res ClinPract 23 : 155-161, 1994

15. Ludvik B, Nolan JJ, Roberts A, Baloga J, Joyce M, Bell JM, Olefsky JM : A noninvasive method to measure splanchnic glucose uptake after oral glucose administration. J Clin Invest 95 : 22322238, 1995

16. Imano E, Kanda $\mathrm{T}$, Ishigami $\mathrm{Y}$, Kubota $\mathrm{M}$, Ikeda M, Matsuhisa M, Kawamori R, Yamasaki $\mathrm{Y}$ : Interferon induces insulin resistance in patients with chronic active hepatitis C. J Hepatol $28: 189-193,1998$

17. DeFronzo RA, Tobin JD, Andres R: Glucose clamp technique : a method for quantifying insulin secretion and resistance. Am J Physiol 237 : E214-E223, 1979

18. Harris JA, Benedict FG : In : A biometric study of basal metabolism in man Carnegie Institute of Washington, Publication No, Washington DC, 1919, 279

19. Yamaguchi $\mathrm{M}:$ Japanese standard tables of food composition, 5th ed. In : Tokyo : Ishiyaku Publishers Inc, 1997

20. Gercia-Compean D, Jaquez-Quintana JO, Gonzalez JA, Maldonado-Garza H : Liver cirrhosis and diabetes : risk factors, pathophysiology, clinical implications and management. World J Gastroenterol 15 : 280-288, 2009

21. Matsuhisa M, Shi ZQ, Wan C, Lekas M, Rodgers CD, Giacca A, Kawamori R, Vranic M : The effect of pioglitazone on hepatic glucose uptake measured with indirect and direct methods in alloxan-induced diabetic dogs. Diabetes 46 : 224-231, 1997

22. Selberg O, Burchert W, vd Hoff J, Meyer GJ, Hundeshagen H, Radoch E, Balks HJ, Müller $\mathrm{MJ}$ : Insulin resistance in liver cirrhosis. Positron-emission tomography scan analysis of skeletal muscle glucose metabolism. J Clin Invest 91 : 1897-1902, 1993
23. Kruszynska Y, Williams N, Perry M, Home P : The relationship between insulin sensitivity and skeletal muscle enzyme activities in hepatic cirrhosis. Hepatology 8 : 1615-1619, 1988

24. Yamanaka H, Genjida K, Yokota K, Taketani Y, Morita K, Miyamoto KI, Miyake H, Tashiro S, Takeda E : Daily pattern of energy metabolism in cirrhosis. Nutrition $15: 749-754,1999$

25. Lautz HU, Selberg O, Korber J, Bürger M, Müller MJ : Protein-calorie malnutrition in liver cirrhosis. ClinInvestig 70 : 478-486, 1992

26. Marchesini G, Pacini G, Bianchi G, Patrono D, Cobelli C : Glucose disposal, beta-cell secretion, and hepatic insulin extraction in cirrhosis : a minimal model assessment. Gastroenterology $99: 1715-1722,1990$

27. Lee $\mathrm{Y}$, Hirose $\mathrm{H}$, Ohneda M, Johnson JH, McGarry JD, Unger RH : Beta-cell lipotoxicity in the pathogenesis of non-insulin-dependent diabetes mellitus of obese rats : impairment in adipocyte-beta-cell relationships. ProcNatl Acad Sci U S A91 : 10878-10882, 1994

28. Yu C, Chen Y, Cline GW, Zhang D, Zong H, Wang Y, Bergeron R, Kim JK, Cushman SW, Cooney GJ, Atcheson B, White MF, Kraegen EW, Shulman GI : Mechanism by which fatty acids inhibit insulin activation of insulin receptor substrate-1 (IRS-1)-associated phosphatidylinositol 3-kinase activity in muscle. J Biol Chem 277 : 50230-50236, 2002

29. Müller MJ, Böker KH, Selberg O : Are patients with liver cirrhosis hypermetabolic? Clin Nutr 13 : 131-44, 1994

30. Sugihara K, Yamanaka-Okumura H, Teramoto A, Urano E, Katayama T, Mori H, Utsunomiya $\mathrm{T}$, Shimada M, Takeda E : Recovery pattern of non-protein respiratory quotient and non-esterified fatty acids after liver resection. Nutrition. 2013 Epub

31. Teramoto A, Yamanaka-Okumura H, Urano E, Nakamura-Kutsuzawa T, Sugihara K, Katayama T, Miyake H, Imura S, Utsunomiya T, Shimada $\mathrm{M}$, Takeda $\mathrm{E}$ : Comparison of measured and predicted energy expenditure in patients with liver cirrhosis. Asia Pac J Clin Nutr. 2014 in press

32. Merli M, Riggio O, Romiti A, Ariosto F, Mango L, Pinto G, Savioli M, Capocaccia L : Basal energy production rate and substrate use in stable cirrhotic patients. Hepatology 12 : 106-12, 1990

33. Sotaniemi EA, Keinänen K, Lahtela JT, Arranto AJ, Kairaluoma M : Carbohydrate intolerance 
associated with reduced hepatic glucose phosphorylating and releasing enzyme activities and peripheral insulin resistance in alcoholics with liver cirrhosis. J Hepatol 1 : 277-290, 1985

34. Nielsen MF, Caumo A, Aagaard NK, Chandramouli V, Schumann WC, Landau BR, Schmitz O, Vilstrup H : Contribution of defects in glucose uptake to carbohydrate intolerance in liver cirrhosis : assessment during physiological glucose and insulin concentrations. Am J Physiol Gastrointest Liver Physiol 288 : 11351143, 2005

35. Hui JM, Sud A, Farrell GC, Bandara P, Byth K, Kench JG, McCaughan GW, George J : Insulin resistance is associated with chronic hepatitis $\mathrm{C}$ and virus infection fibrosis progression. Gastroenterology $125: 1695-1704,2003$

36. Fartoux L, Poujol-Robert A, Guéchot J, Wendum D, Poupon R, Serfaty L: Insulin resistance is a cause of steatosis and fibrosis progression in chronic hepatitis C. Gut 54 : 1003-8, 2005
37. Imano E, Kanda T, Nakatani Y, Motomura M, Arai K, Matsuhisa M, Yamasaki Y, Hori M : Imapaire splanchnic and peripheral glucose uptake in liver cirrhosis. J Hepatol 31 : 469-473, 1999

38. Kruszynska YT, Meyer-Alber A, Darakhshan $\mathrm{F}$, Home PD, McIntyre $\mathrm{N}$ : Metabolic handling of orally administered glucose. J Clin Invest 91 : 1057-1066, 1993

39. Meyer-Alber A, Hartmann H, Stumpel F, Creutzfeldt W : Mechanism of insulin resistance in CCl4-induced cirrhosis of rats. Gastroenterology $102:$ :223-229, 1992

40. Del Prato S, Bonadonna RC, Bonora E, Gulli G, Solini A, Shank M, De Fronzo RA : Characterization of cellular defects of insulin action in type 2 (non-insulin-dependent) diabetes mellitus. J Clin Invest 91 : 484-493, 1993

41. Petrides AS, DeFronzo RA : Failure of glucagon to stimulate glycogenolysis in well-nourished patients with mild cirrhosis. Metabolism 43 : 85-89, 1994 